\title{
Hybrid Carbon Dioxide Heat Pump for the Multifamily Residential Buildings in the Heat Supply System Based on CHP
}

\author{
Sit M.L., Juravliov A.A. \\ Institute of Power Engineering \\ Kishinau, Republic of Moldova
}

\begin{abstract}
The work is devoted to centralized heat supply systems based on CHP plants and the use with them heat pumps (HP) on carbon dioxide as refrigerant. Heat pumps are used in heat supply systems for buildings and use the heat of the outside air and, at the same time, the heat of the return network water (WWR) as a source of low-grade heat (LHP). The aim of the study is to develop a structural diagram of such a heat pump, where the outside air is heated by a heat exchanger installed in the return water line of the heating system, to develop a hydraulic circuit of a heat pump taking into account the law of regulation of the building heating system, to develop an algorithm for controlling the operating modes of the so-called balancing heat exchanger installed after gas cooler and internal heat exchanger of the heat pump. The most significant results were the hydraulic circuit of the heat pump, the aerodynamic circuit of the air supply path to the heat pump evaporator, the balancing heat exchanger control system, taking into account the requirement to ensure the operation of the control valve in a single-phase flow. The significance of the results obtained consisted in obtaining the dependences between the CO temperature graph and the parameters of the thermodynamic cycle of the heat pump, which ensured the operation of the control valve of the heat pump in a single-phase environment.

Keywords: carbon dioxide heat pump, district heating, automatic control.

DOI: https://doi.org/10.52254/1857-0070.2021.3-51.08

UDC: 697.34; 621.577.42
\end{abstract}

Pompa de căldură hibridă cu dioxid de carbon pentru utilizarea în clădiri de locuit multietajate în sistemul de termoficare cu CET

Sit M.L., Juravleov A.A.

Institutul de Energetică

Chişinău, Republica Moldova

Rezumat. Lucrarea este dedicată sistemelor centralizate de alimentare cu căldură în baza centralelor de cogenerare și utilizării pompelor de căldură (HP) în acestea pe dioxid de carbon. Pompele de căldură sunt utilizate în sistemele de alimentare cu căldură pentru clădiri și folosesc căldura aerului exterior și, în același timp, căldura apei din rețeaua de retur (ARR) ca sursă de căldură cu potențial termic scăzut (PTS). Scopul studiului este: de a dezvolta o schemă structurală a unei astfel de pompe de căldură, în care aerul exterior este încălzit de un schimbător de căldură instalat în linia de retur a apei din sistemul de încălzire, de a dezvolta un circuit hidraulic al unei pompe de căldură luând în considerare legea reglării unui sistem de încălzire a clădirilor, de a dezvolta un algoritm pentru controlul modurilor de funcționare ale aşa-numitului schimbător de căldură de echilibrare instalat după răcitorul de gaz și schimbătorul de căldură intern al pompei de căldură. Cele mai semnificative rezultate sunt elaborarea circuitului hidraulic al pompei de căldură, circuitului aerodinamic al căii de alimentare cu aer către evaporatorul pompei de căldură, sistemul de control al schimbătorului de căldură de echilibrare, ținând cont de cerința de a asigura funcționarea supapei de control într-un flux monofazat. Semnificația rezultatelor obținute constă în obținerea dependentelor dintre graficului de temperatura a sistemului de încălzire alimentat de pompa de căldură și parametrii ciclului termodinamic al pompei de căldură, care asigură funcționarea supapei de control a pompei de căldură într-un mediu monofazat.

Cuvinte-cheie: pompa de căldură cu dioxid de carbon, încălzire, sistem de control al pompei de căldură, ciclu termodinamic.

Гибридный тепловой насос на диоксиде углерода для многоквартирных жилых зданий в системе теплоснабжения на базе ТЭЦ

Шит М.Л., Журавлев А.А.

Институт энергетики, Кишинев, Республика Молдова

Аннотация. Работа посвящена системам централизованного теплоснабжения на базе ТЭЦ и использованием в них тепловых насосов (ТН) на диоксиде углерода. Тепловые насосы использованы в системах теплоснабжения зданий и используют в качестве источника низкопотенциальной теплоты (НПТ) теплоту наружного воздуха, и, одновременно, теплоту обратной сетевой воды (ОСВ). Целью исследования является разработка структурной схемы такого теплового насоса, где наружный воздух подогревается 
теплообменником, установленным в лини обратной сетевой воды системы теплоснабжения, разработка гидравлической схемы теплового насоса с учетом закона регулирования системы отопления здания, разработка алгоритма управления режимами работы так называемого балансирующего теплообменника, установленного после газоохладителя и внутреннего теплообменника теплового насоса. Наиболее существенными результатами являются гидравлическая схема теплового насоса, аэродинамическая схема тракта подачи воздуха на испаритель теплового насоса, система управления балансирующим теплообменником с учетом требования обеспечения работы регулирующего вентиля в однофазном потоке. Значимость полученных результатов состоит в получении зависимостей между температурным графиком СО и параметрами термодинамического цикла теплового насоса, при которых обеспечивается работа регулирующего клапана теплового насоса в однофазной среде. Установлено, что в схему этой САР должен быть включен функциональный преобразователь, связывающий температуру наружного воздуха координаты выхода балансирующего теплообменника на термодинамическом цикле теплового насоса. Разработана структурная схема регулирования режима теплового насоса посредством включения после газоохладителя и первичного контура внутреннего теплообменника так называемого балансирующего теплообменника. Режим работы этого теплообменника выбирается из условия обеспечения режима работы регулирующего клапана теплового насоса только на однофазной среде. Разработана структурная схема системы автоматического регулирования значения величины перепада энтальпий на этом теплообменнике.

Ключевые слова: тепловой насос на диоксиде углерода, отопление, система управления тепловым насосом, термодинамический цикл.

\section{ВВЕДЕНИЕ}

Вопросы применения тепловых насосов в системах теплоснабжения с ТЭЦ многоэтажных, многоквартирных домов освещены в ряде публикаций [1-6]. Так, в [7-9] предлагается использование ТН непосредственно на ТЭЦ, в [4,5] предлагается использование только крупного ТН и подключенных к нему, так называемых поддерживающих тепловых насосов. Нами предлагается использование ТЭЦ совместно с местными тепловыми насосами, получающими НПТ от обратной сетевой воды и от окружающего воздуха (публикации авторов в данном журнала за 2020 год).

Глобальная цель исследований, проводимых в данной работе: разработка схемного технического решения системы «ТЭЦ-тепловые насосы» для обеспечения отопления зданий при условии снижения расхода газа на ТЭЦ и снижения выбросов $\mathrm{CO} 2$.

Поставленную цель планируется достичь за счет решения задачи интеграции ТН в тепловую схему системы теплоснабжения с ТЭЦ посредством блока связи системы теплоснабжения (СТC) с ТН. Блок связи СТС с ТН передает теплоту наружного воздуха и часть теплоты обратной сетевой воды (OCB) испарителю теплового насоса.

Известно, что температура обратной сетевой воды (ОСВ) зависит от многих факторов, таких как температура и расход сетевой воды, режим регулирования отопительной нагрузки, свойства теплового ограждения дома, метеоусловия. В данной работе предлагается использовать наружный воздух, который проходит через дополнительный теплообменник, установленный в линии обратной сетевой воды. Для теплового насоса на диоксиде углерода - это диапазон температур от $5^{\circ} \mathrm{C}$ до $20^{\circ} \mathrm{C}$.

\section{МЕТОДЫ, РЕЗУЛЬТАТЫ И ОБСУЖДЕНИЕ}

Нами предлагается к рассмотрению следующая схема системы теплоснабжения с ТЭЦ (СНР) (рис.1,2). На рис.1 приведена схема подключения здания, снабженного тепловым насосом через теплообменник «вода-воздух», обозначенным на рис. 1 поз.4, и установленным в обратном трубопроводе сети теплоснабжения. Наружный воздух подогревается в этом теплообменнике и поступает на испаритель теплового насоса НP. В данной схеме теплообменник HEX выступает в качестве звена, суммирующего теплоту наружного воздуха и теплоту, отбираемую от обратной сетевой воды. Пусть наружный воздух имеет температуру $0^{\circ} \mathrm{C}$, и воздух, подогретый в теплообменнике «водавоздух», поз.4, рис.2. имеет температуру $20^{\circ} \mathrm{C}$. Тогда этот подогретый воздух вызывает испарение $\mathrm{CO} 2$ в испарителе, поз.7, при $15^{\circ} \mathrm{C}$.

На рис.2 цифрами черного цвета обозначены точки на Н-р диаграмме. Т.4 температуру хладагента после переохладителя 
газа, поз. 4, т.8. определяет режим работы внутреннего теплообменника, поз.2.

$$
h_{13}-h_{12}=h_{9}-h_{10} . h_{2}-h_{6}=h_{3}-h_{7} .
$$

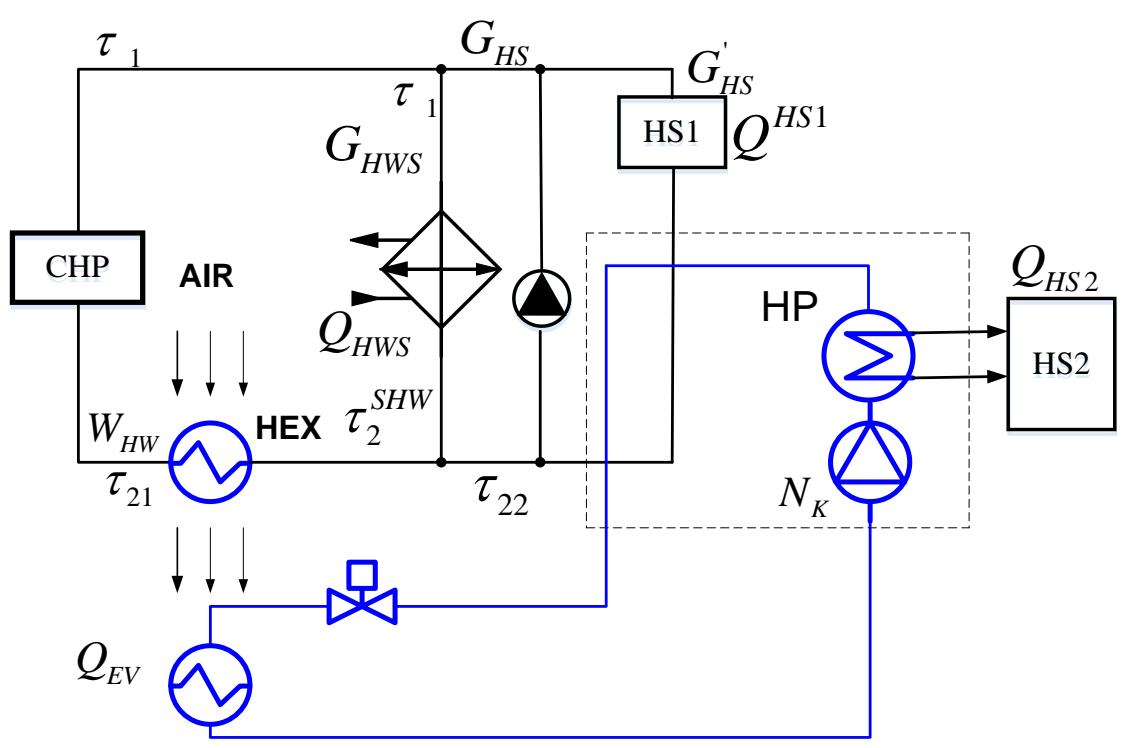

HS1- система отопления существующего здания, HS2- система теплоснабжения нового здания, HPтепловой насос; HЕX (поз. 11, см. рис.2) - теплообменник отбора теплоты от системы теплоснабжения, СНР- ТЭЦ, $Q_{H W S}$ - система ГВС здания.

Рис.1. Схема включения теплового насоса в систему теплоснабжения с ТЭЦ. ${ }^{1}$

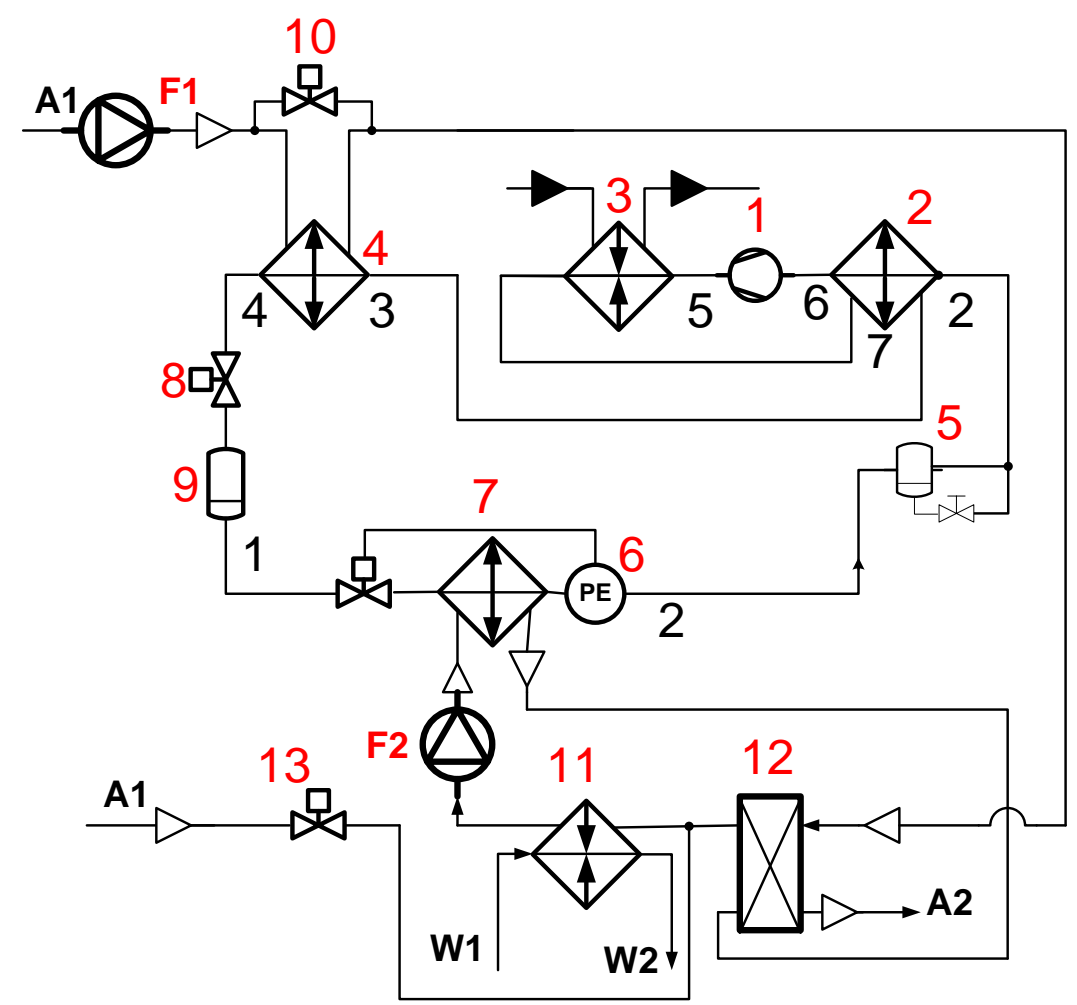

Теплообменник поз.4, входной и выходной потоки W1, W2 испаритель теплового насоса, поз.7. F1 вентилятор для съема теплоты с теплообменника 10, F2 - вентилятор испарителя, 12- рекуператор, А1наружный воздух, А2 - воздух, удаляемый после прохождения через испаритель и рекуператор, 13 заслонка.

Рис.2. Гидравлическая схема теплового насоса и его узла связи с системой теплоснабжения. ${ }^{2}$ 
Рекуператор 12 может отсутствовать в схеме рис.2. Тогда снизится мощность вентилятора F1.

На рис.3 точки 6 и 5, 11 и 10 соединяют изоэнтропы со значением коэффициента 0.7 . На диаграмме т.7 определяет температуру хладагента после газоохладителя, поз.3, т.5 температуру хладагента после компрессора, поз.1. Заслонка поз.13, рис.2, регулирует расход воздуха, подаваемого вентилятором испарителя теплового насоса.

Расход воздуха, подаваемого на испаритель, вычисляется с использованием следующей формулы:

$$
\begin{aligned}
& G_{A 4} c_{A}\left(T_{E V}-T_{A 4}\right)+G_{E V} c_{A}\left(T_{A 11 I N}-T_{A 11 O U T}\right)= \\
& =G_{C O 2}\left(h_{1}-h_{2}\right)
\end{aligned}
$$

В таблице 1 и на рис. 2 приведены данные термодинамического цикла для температурного графика системы отопления $80 / 30$ при расчетной температуре внутреннего воздуха $21^{\circ} \mathrm{C}$ и расчетной температуре наружного воздуха минус $16^{\circ} \mathrm{C}$.

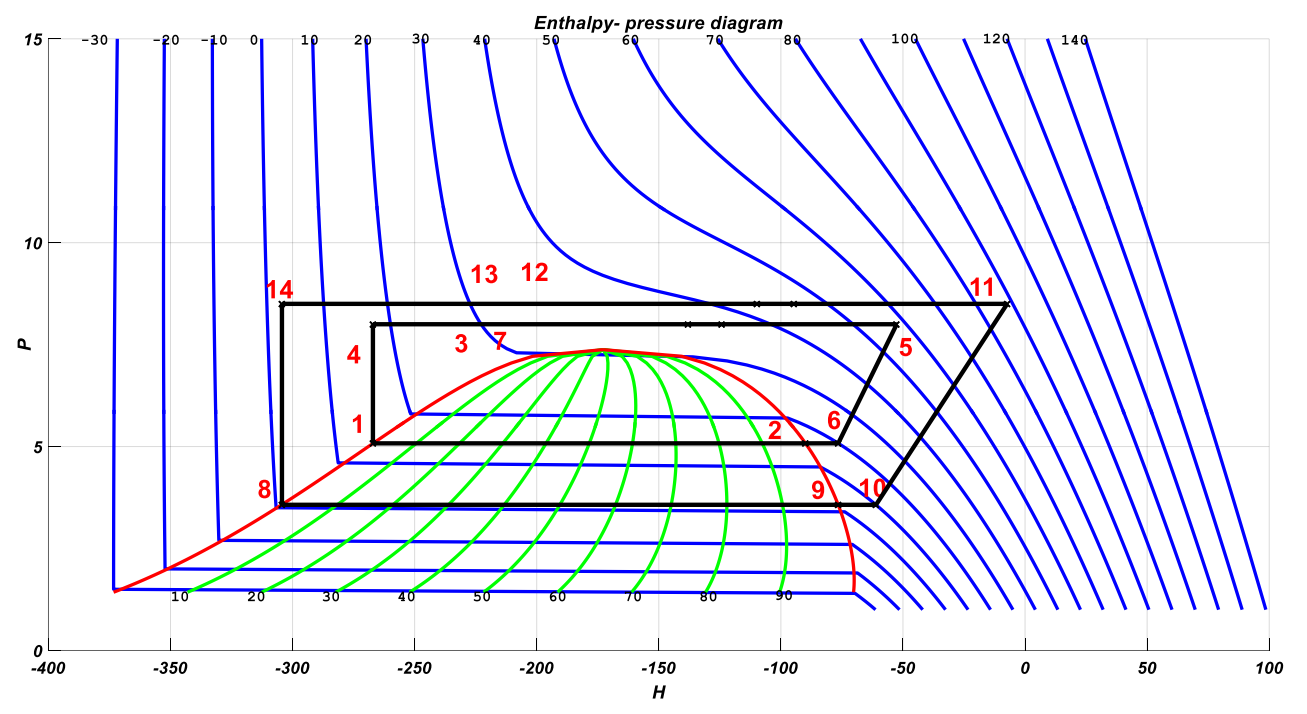

Рис.3. Термодинамический цикл теплового насоса в Н-р координатах. ${ }^{3}$

Таблица 1. Значения теплофизических параметров термодинамического цикла. ${ }^{4}$

\begin{tabular}{|l|l|l|l|l|l|}
\hline & $\mathrm{T}, \mathrm{K}$ & $\begin{array}{l}\mathrm{P}, \\
\mathrm{MPa}\end{array}$ & $\begin{array}{l}\% \\
\mathrm{G}\end{array}$ & $\mathrm{H} \mathrm{kJ} / \mathrm{kg}$ & $\begin{array}{l}\mathrm{S} \\
\mathrm{kJ} / \mathrm{kg}\end{array}$ \\
\hline 1 & 15.00 & 5.08 & 0 & -267.89 & -1.604 \\
\hline 2 & 15.00 & 5.08 & 100 & -89.936 & -0.989 \\
\hline 3 & 28.00 & 8.00 & 0 & -232.542 & -1.499 \\
\hline 4 & 17.59 & 8.00 & 0 & -267.089 & -1.616 \\
\hline 5 & 58.04 & 8.00 & 100 & -52.556 & -0.921 \\
\hline 6 & 20.00 & 5.08 & 100 & -76.509 & -0.943 \\
\hline 7 & 31.00 & 8.00 & 0 & -217.052 & -1.448 \\
\hline 8 & 1.00 & 3.57 & 0 & -304.348 & -1.731 \\
\hline 9 & 1.00 & 3.57 & 100 & -76.553 & -0.899 \\
\hline 10 & 10.00 & 3.57 & 100 & -61.061 & -0.843 \\
\hline 11 & 88.47 & 8.50 & 100 & -7.324 & -0.798 \\
\hline 12 & 35.00 & 8.50 & 0 & -197.233 & -1.386 \\
\hline 13 & 32.00 & 8.50 & 0 & -218.163 & -1.454 \\
\hline 14 & 2.83 & 8.50 & 0 & -304.348 & -1.749 \\
\hline
\end{tabular}

Расчетное значение СОР при температуре наружного воздуха составляет:

$$
\begin{aligned}
& C_{0 C}=\frac{h_{5}-h_{7}}{h_{5}-h_{6}}= \\
& =\frac{(-52.556-(-267.089)) \cdot 0.92}{-52.556-(-76.509)}=6.9
\end{aligned}
$$

Значение расчетного СОР при минус $16^{\circ} \mathrm{C}$ составляет:

$$
\begin{aligned}
& \operatorname{COP}_{-16 C}=\frac{h_{11}-h_{12}}{h_{11}-h_{10}}= \\
& =\frac{(-7.324-(-197.233)) \cdot 0.92}{(-7.324)-(-61.061)}=3.2 .
\end{aligned}
$$

Где $\eta_{m e}=0.92 \quad-\quad$ кпд электропривода компрессора. Если предположить, что электрическая мощность вентиляторов 
балансирующего теплообменника (ТБР), поз. 4 , и испарителя, рис. 2 составит $20 \%$ от его тепловой мощности (что соответствует данным каталогов фирм - изготовителей драйкулеров), то значения СОР составят соответственно: $C O P_{0 C} \approx 6$, а $C O P_{-16} \approx 2.8$.

Отрезок диаграммы 5-7 характеризует теплоту, отдаваемую газоохладителем, отрезок 7-3 - характеризует перепад энтальпии на внутреннем газоохладителе, 3-4 падение энтальпии на переохладителе газа 4. Переохладитель газа 4 обеспечивает режим работы испарителя, при котором газ вначале подогревается в переохладителе 4, затем в рекуператоре, а лишь потом, пройдя через эти два аппарата, газ поступает к испарителю. Координата т.4 выбирается из условия, чтобы, до входа на линию насыщения, регулирующую заслонку, поз. 9, находился в условиях течения через него однофазной среды. Изоэнтропический коэффициент компрессора принят равным 0.7. Точки 9, 10, 12,13 термодинамического цикла характеризуют внутренний теплообменник. Точки 1 и 4, 8 и 4 описывают изоэнтальпы, характеризующие работу регулирующего клапана 8. Внутренний четырехугольник на рис.3 показывает термодинамический цикл при температуре наружного воздуха в $0^{\circ} \mathrm{C}$, а внешний четырехугольник цикл при температуре наружного воздуха минус $18^{\circ} \mathrm{C}$.

Критерием качества работы теплового четырехполюсника является обеспечение передачи расчетной, по условию реализации температурного графика, тепловой мощности в нагрузку.

Для системы управления режимом работы газоохладителя возмущениями являются: отклонения значений величин температуры обратной сетевой воды от значений наружной температуры воздуха, температуры газа после компрессора.

Запишем уравнение теплового баланса газоохладителя.

$$
G_{\text {СOMPR }} c_{C O 2}\left(T_{8}-T_{4}\right)=G_{A E R} c_{a e r}\left(T_{A E R_{-} O}-T_{A E R}\right)
$$

и условие $h_{1}=h_{4}$ (линия 4-1 - является изоэнтальпой, и т.4 лежит на пограничной кривой (линии насыщения)), которое рассматривается совместно с уравнением (2).

Уравнение теплового баланса теплообменника поз.11 (рис.2), установленного в линии обратной сетевой воды системы теплоснабжения:

$$
G_{\text {AER }}\left(T_{\text {aer } 4 \text { out } 2}-T_{\text {aer } 4 \text { out }}\right)=G_{\text {WAT }}\left(T_{\text {WIN }}-T_{\text {WOUT }}\right)
$$

Для системы управления режимом работы теплообменника поз.11 (рис.2), установленного в линии обратной воды, возмущениями являются температура воздуха после первого по ходу воздуха теплообменника, температура воды на входе в теплообменник. Для стабилизации перепада температур воды на теплообменник, поз.11, необходимо изменять длину этого теплообменника при постоянных расходах теплоносителей или регулировать режим работы обводом части потока вокруг теплообменника, поз.4. Сравним оба режима. Критерием качества работы является обеспечение режима работы испарителя ТН при котором его тепловая мощность обеспечивает заданный режим работы теплового насоса.

Рекуператор должен быть спроектирован из условия пропуска через себя тепловой мощности, равной максимально мощности испарителя при расчетной температуре наружного воздуха. Тепловой процесс в рекуператоре R1 удовлетворяет уравнению:

$$
T_{\text {linr }}-T_{\text {loutr }}=T_{2 \text { inr }}-T_{2 \text { outr }}
$$

Рекуператор рассматриваем как звено, не подверженное действию возмущений. Рекуператор (4) рассматриваем как инерционное звено с запаздыванием со входом $T_{\text {linr }}$ и выходом $T_{2 o u t r}$, где передаточная функция между входом и выходом имеет вид [18]:

$$
\frac{t_{2 o u t r}(p)}{t_{\text {linr }}(p)}=W_{R}(p)=b(p)\left(1-e^{-\frac{\tau}{a(p)}}\right)
$$

Рассмотрим динамический режим работы этих трех теплообменников при постоянных и случайных возмущениях и выполнении условия обеспечения подвода заданной тепловой мощности к испарителю теплового насоса.

Поскольку $T_{A E R}$ для рассматриваемой задачи является задающей величиной, определяющей все параметры цикла, то необходимо 
корректировать коэффициент усиления регулятора С2 с учетом уравнения статики (2). Подвод заданной тепловой мощности к испарителю обеспечивается за счет управления: регулирующими клапанами поз. 6, 8 (рис.1), и производительностью компрессора поз.1. Давление на входе в испаритель обеспечивается за счет уставок этих регулирующих клапанов. Перепад температур между контурами испарителя обеспечивается за счет выбора режимов работы теплообменника поз. 11.

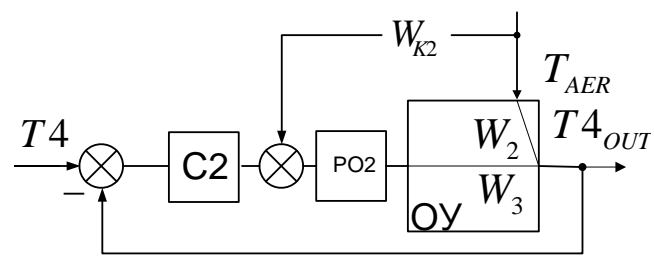

C2- регулятор, РО2- регулирующий орган, $W_{2}, W_{3}, W_{K 2}-$ передаточные функции, ОУ-объект управления.

Рис.4. Структурная схема САУ теплообменником балансировки режима ${ }^{5}$.

Регулирование значения величины координаты т.4 термодинамического цикла (рис.2) обеспечивается за счет регулирования производительности вентилятора F1 ТБР, поз.4, или байпасирования через заслонку 9 (рис.2). При изменении расхода вентилятора F1 теплообменника балансировки режима, поз. 4, может изменяться температура воздуха после теплообменника балансировки режима.

$$
h_{4}=G_{A F} \frac{T_{\text {aer_out } 4}-T_{A E R}}{G_{C O 2}}+h_{3} .
$$

$G_{A F}-$ расход воздуха через теплообменник 4. Уравнение (6) описывает статическое значение управляющего воздействия в САУ управления координатой т.4. Передаточная функция между выходной величиной (энтальпией диоксида углерода в.4. или, что тоже самое температурой $\mathrm{CO} 2$ в т.4) и расходом через заслонку 9.

В качестве динамической модели, связывающей расход воздуха и температуру хладагента на выходе теплообменника, поз.4, применим описанную в [15, с.103]

$$
W_{T 4-G}=\frac{k_{1}}{a_{1} p^{2}+a_{2} p+a_{3}}
$$

Для стабилизации режима работы испарителя при данной температуре наружного воздуха необходимо обеспечить при заданном расходе воздуха через него соответствующую температуру воздуха путем регулирования расхода сетевой воды через теплообменник поз.11.

Структурная схема САУ режимом работы испарителя может иметь вид каскадной системы автоматического регулирования, в которой внутренний контур обеспечивает стабилизацию заданной величины температуры воздуха, а наружный контур представляет собой регулятор тепловой мощности воздуха. Во внутреннем контуре температура воздуха стабилизируется за счет изменения расхода через теплообменник 11, например, путем байпасирования расхода сетевой воды вокруг него. Для регулирования тепловой мощности, подаваемой на испаритель необходимо регулировать степень открытия заслонки 13 , рис. 2 .

\section{ЗАКЛЮЧЕНИЕ}

1. Применение теплового насоса, использующего для отопления здания теплоту обратной сетевой воды и наружного воздуха является рациональным с точки зрения экономии топлива на ТЭЦ.

2. Для климатических условий Республики Молдова расчетный СОР такого теплового насоса оказывается выше 7 при температурном графике системы теплоснабжения здания, отапливаемого тепловым насосом 80/30.

3. Для надежного регулирования режима работы такого теплового насоса необходимо включение в его структуру теплообменника «воздух - хладагент» для охлаждения хладагента выходящего из газооохладителя до температуры, при которой регулирующий клапан теплового насоса будет работать на однофазной среде.

\section{APPENDIX 1 (ПРИЛОЖЕНИЕ 1)}

${ }^{1}$ Figure 1. HS1- heating system of the building, HS2heat supply system of the new building, HP- heat pump; HEX (pos. 11, see fig. 2) - heat exchanger for extracting heat from the heat supply system, CHPCHP, the building's hot water supply system.

${ }^{2}$ Figure 2. Hydraulic diagram of the heat pump and his communication unit with the heat supply system (heat exchanger pos. 4, flows W1, W2) with the evaporator of the heat pump, pos. 7, ((flows T1, T2). F1 - fan for 
removing heat from the heat exchanger 10, F2 - evaporator fan, 12 - recuperator, A1 - outside air, A2 - air removed after passing through the evaporator and recuperator.

3, Figure 3. Thermodynamic cycle of a heat pump in H-p coordinates.

${ }^{4}$ Table 1. Values of thermophysical parameters of the thermodynamic cycle.

${ }^{5}$ Figure 4. C2- controller, PO2- control valve, $W_{2}, W_{3}, W_{K 2}-$ transfer functions. Block diagram of automatic control system with heat exchanger for balancing mode, $\mathrm{OY}$-controlled object.

\section{БЛАГОДАРНОСТЬ}

Результаты были получены в рамках проекта Госпрограммы с номером 20.80009.7007.18: «Экоинновационные технические решения по снижению энергопотребления зданий и разработка опций по развитию интеллектуальных сетей с высокой интеграцией ВИЭ в Молдове»

\section{ЛИТЕРАТУРА (REFERENCES)}

[1] Lund H, Werner S., Wiltshire R., Svendsen S., Thorsen J.E., Hvelplund F., et al. $4^{\text {th }}$ Generation District Heating (4GDH): integrating smart thermal grids into future sustainable energy systems. Energy 68(2014) 1-11. http://dx.doi.org/10.1016/j.energy.2014.2.089.

[2] Rama M., Wahlroos M. Introduction of new decentralised renewable heat supply in an existing district heating system. Energy 154 (2018) 68-79, https://doi.org/10.1016/j.energy.2018.03.105

[3] Ommen, T. S. (2015). Heat Pumps in CHP Systems: High-efficiency Energy System Utilizing Combined Heat and Power and Heat Pumps. DTU Mechanical Engineering. DCAMM Special Report, No. S187.

https://backend.orbit.dtu.dk/ws/portalfiles/portal/ 118855919/S187_Torben_S._Ommen.pdf (accessed 12.12.2020).

[4] Ommen, T., Markussen, W.B., Elmegaard B. Heat pumps in combined heat and power systems. Energy 2014; 76:989-1000. doi:10.1016/j.energy. 2014.09.016.

[5] Elmegaard B., Ommen T., Markussen M., Iversen, J. Integration of space heating and hot water supply in low temperature district heating. Energy \& Buildings 2015; doi: 10.1016/j. enbuild.2015.09.003.Management 50, 19911999.

[6] Lo Basso G, Nastasi B.,Salata F., Golasi J. Energy retrofitting of residential buildings-How to couple Combined Heat and Power (CHP) and Heat Pump (HP) for thermal management and off-design operation. Energy and Buildings 151 (2017) 293-305.

http://dx.doi.org/10.1016/j.enbuild.2017.06.060.
[7] https://www.districtenergy.org/HigherLogic/System/DownloadDocumentFile.ashx?Document-

FileKey=3a4ccdd4-ac77-2489-c35a7e9dcb8b2e64 (accessed 12.08.2012).

[8] Getnet Tadesse Ayele, Mohamed Tahar Mabrouk, Pierrick Haurant, Björn Laumert, Bruno Lacarrière. Optimal placement and sizing of heat pumps and heat only boilers in a coupled electricity and heating networks. Energy, Elsevier, 2019, 182, pp.122134.10.1016/j.energy.2019.06.018.hal-02152378.

[9] Vinnemeier Ph., Virsum M., Malpiece D., Bove R. Integration of heat pumps into thermal plants for creation of large-scale electricity storage capacities. Applied Energy, 184(2016), p.506-522.

[10]Lavrov N.A. Mnogourovnevaia Sistema modelirovania nestatsionarnih I meniauschihsia rejimov raboti nizkotemperaturnih ustanovok. [Multilevel system of modeling nonstationary and variable operating modes of low-temperature installations]. Moscow, 2013. Doctor thesys. https://www.google.com/url?sa=t\&rct=j\&q=\&esr $\mathrm{c}=\mathrm{s} \&$ source $=$ web $\& \mathrm{~cd}=3 \& \mathrm{cad}=\mathrm{rja} \& u a c t=8 \& \mathrm{ved}=$ 2ahUKEwjh9rD26p7fAhXH2KQKHeTPDfEQFjACegQIA-

BAC\&url=http $\% 3 \mathrm{~A} \% 2 \mathrm{~F} \% 2 \mathrm{Fwww}$. bmstu.ru\%2F ps $\% 2 \mathrm{~F} \sim$ lavrov $\% 2 \mathrm{Ffileman} \% 2 \mathrm{Fdown}-$ load $\% 2 \mathrm{~F} \% 25 \mathrm{D} 0 \% 259 \mathrm{~B} \% 25 \mathrm{D} 0 \% 25 \mathrm{~B} 0 \% 25 \mathrm{D} 0 \%$ 25B2\%25D1\%2580\%25D0\%25BE\%25D0\%25B 2-\%25D0\%25B4\%25D0\%25B8\%25D1\%2581\% 25D1\%2581.pdf\&usg=AOv-

Vaw0RUNk 0AuFo6c2en0a7Lg0 (accessed 14.08.2021).

[11]Redko A.A., Taradai A.M., Chernokriluik V.V., Esin T.S. Kombinirovannie sistemi teplosnabjenia s vozobnovliaemimi istochnikami tepla. [Parameters of a Heat Supply System at a Lower Temperature Chart], Enegrosberejenie, energe-tika, energoaudit [Energy saving, energetics, energy audit]. 199(29)2014, p.42-46. http://eee.khpi.edu.ua/article/view/33556/30109 , accessed 19.08.2021.

[12] Ogurechnikov L.A. Resursosberegaiuschaia kombinirovannaia sistema teplosnabjenia. [Resources saving combined heat supply system]. https://vodapol.ru/Arts/Resursosberegayushaya_sistema.php, accessed 19.08.2021.

[13] Musabekov R.A., Abilidinova S.K., Rasmuhametova A.S. Effektivnosti system centralizovannogo teplosnabjenia $\mathrm{v}$ usloviah sovmestnogo ispolizovania teolovih nasosov. [Efficiency of district heating systems under conditions of joint usage of heat pumps]. Vestnik AUES [Herald of AUEC] Almaty: AUEC, 2017 - №1(36). - p.5-19.

[14] Nozdrenko G.V. Komplexnii exergeticheskii analiz energoblokov TES s novimi technologiami [Comlex ezergetic analysis of CHP with new technologies] Novosibirsc, 2009. 
[15] Frantseva A.A. Optimizatsionnie issledovania TETS s gazosetevimi podogrevateliami. [Optimization studies of thermal power plants with a gas network heater and freon thermotransformers]. Novosibirsk, 2015. https://нгту.pф/files/dissertations/dissertaciya franceva 142916052027.pdf (accessed 21.08.2021).

[16]Bejan P.I. Spravochnik po teploobmennim apparatam. [Heat Exchanger Handbook], Mashinostroienie, Moscow, 1989.

[17] Derevich E.G., Smirnova E.G. Calculating the Parameters of Heat Transfer between Countercurrent Flows with Variable Thermophysical Properties. Theoretical Foundations of Chemical Engineering, Vol. 36, No. 4, 2002, pp. 341-345.

[18]Dudnikov E.G. Avtomaticheskoe upravlenie v himicheskoi promishlennosti. [Automatic control in chemical industry]. Mashinostroenie, 1987. 368 p.

[19] Automatic control of a heat exchanger with changing operation conditions. http://www.mathematik.tu-dortmund.de/papers/BayazitBicerKulaliMueminogluTorres2008.pdf (accessed 12.08.2021).

[20] Serov E.P., Korolikov B.P. Dinamika parogenerwtorov [Steam generators dynamics]. Moscow, 1972 Energia, 1972.

[21]Applying heat exchanger control strategies. https://www.controleng.com/articles/applyingheat-exchanger-control-strategies/ (accessed 11.08.2021).

[22] Saji K. S. and Sasi Kumar M. PI-Controller Tuning for Heat Exchanger with Bypass and Sensor. International Journal of Electrical Engineering. ISSN 0974-2158 Volume 5, Number 6 (2012), pp. 679-689.

[23] Fisher m., Nelles O., Isermann R. Adaptive predictive control of a heat exchanger based of fuzzy model. Control Engineering Practice 6 (1998) 259-269.

[24] Shinskey G.F. PID dead - time control of distributed processes. Control Engineering Practice 9 (2001) 1177-1183.

\section{Сведения об авторах.}

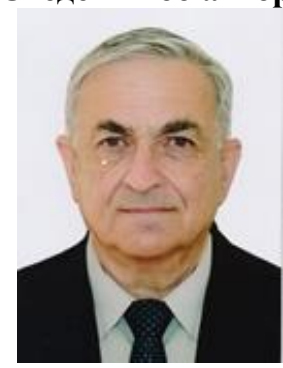

Шит Михаил Львович к.т.н., доцент-исследователь, в.н.с. Область научных интересов: тепловые насосы, автоматическое управление технологическими процессами.

E-mail: mihail_sit@mail.ru
[25] Pangborn H.C., Alleyne A.G. Switched linear control for refrigerant superheat recovery in vapor compression systems. Control Engineering Practice 57(2016)142-156.

[26] Narendra K. S. and George K., "Adaptive control of simple nonlinear systems using multiple models," in Proceedings of the American Control Conference (ACC '02), pp. 1779-1784, Anchorage, AK, USA, May 2002.

[27] Kanellakopoulos I., Kokotovic P. V., and Morse A. S., "Systematic design of adaptive controllers for feedback linearizable systems," IEEE Transactions on Automatic Control, vol. 36, no. 11, pp. 1241-1253, 1991.

[28] Nakanishi J, Farrell J. A., and Schaal S, “Composite adaptive control with locally weighted statistical learning," Neural Networks, vol. 18, no. 1, pp. 71-90, 2005. 2004

[29] Ovsiannik A.V., Matsko I.I., Rychter O.L., Bobovich S.O. Perspektivi primennenia teplonasosnih tehnologii d teplofikatsionnom kompexe goroda Gomelia [Prospects for the use of heat pump technologies in the heating complex of the city of Gomel]. $\quad$ https://elib.gstu.by/bitstream/handle $/ 220612 / 10175 / \%$ D0\%9E\%D0\%B2\%D1\%81 \%D1\%8F\%D0\%BD\%D0\%BD\%D0\%B8\%D0\% BA\%2C\%20\%D0\%90.\%20\%D0\%90.\%20\%D0 \%9F\%D0\%B5\%D1\%80\%D1\%81\%D0\%BF\%D0 $\% \mathrm{~B} 5 \% \mathrm{D} 0 \% \mathrm{BA} \% \mathrm{D} 1 \% 82 \% \mathrm{D} 0 \% \mathrm{~B} 8 \% \mathrm{D} 0 \% \mathrm{~B} 2 \% \mathrm{D}$ $1 \% 8 \mathrm{~B} \ldots$ pdf? sequence $=1 \&$ isAllowed $=\mathrm{y}$ (accessed 11.08.2021).

[30] Rony R.U., Yang H., Krishnan S., Song J. Recent Advances in Transcritical CO2 (R744) Heat Pump System: A Review. Energies 2019, 12, 457; https://doi.org:10.3390/en12030457.

[31] Ma Yitai, Liu Zhongyan, Tian Hua A review of transcritical carbon dioxide heat pump and refrigeration cycles. Energy. 55 (2013), p.156-172.

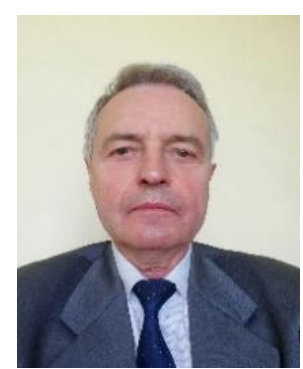

Журавлев Анатолий Александрович - к.т.н., в.н.с. Область научных интересов: микропроцессорные системы управления, промышленная автоматика.E-mail: AZhur5249@mail.ru 